\title{
Plasma levels of tricyclics and related antidepressants: are they necessary or useful?
}

\author{
David Taylor and Denise Duncan
}

Opinions on the value of measuring tricyclic plasma levels vary substantially. In the United States, plasma levels of several tricyclics are frequently used to 'optimise' therapy. In the UK, plasma level monitoring is less often undertaken and measured levels are more usually used simply to assure compliance rather than as a guide to dose adjustment.

The value of antidepressant serum levels was reviewed by a 1985 American Psychiatric Association Task Force (Glassman et al, 1985). This group found that there was an accepted, well defined therapeutic range only for nortriptyline (see table below); there was little agreement on other drugs. Much has been made of the apparent shape of doseresponse curves (sigmoidal, U-shaped, etc.) for several antidepressants. Discussions of this sort seem premature considering the dearth of information supporting any form of dose-response relationship.

Several criteria must be met for plasma level monitoring of any drug to be worthwhile. For example, there should be a wide variation in individual drug handling, clinical response must be difficult to establish in individual patients and plasma levels must relate directly to clinical and adverse effects. Certainly it is well known that plasma tricyclic levels vary considerably in individuals given the same dose, so the first of these criteria is met. However, the second is not: clinical response to antidepressants is readily observed, either using recognised evaluation scales or by subjective evaluation. Thus if a patient fails to respond, the next step is to increase the dose or change the drug: a plasma level measurement is not usually indicated. The third criterion listed is, as we have seen, only partly satisfied: few studies have established a direct relationship between plasma level and efficacy of antidepressants and even where there are studies, the therapeutic range is often poorly defined (see Table 1). Some adverse effects (e.g. CNS and CVS toxicity) do seem, however, to be related to plasma levels (Preskorn \& Fast, 1991). Thus it appears that monitoring of tricyclic plasma levels is of little practical use. Nevertheless, there are aspects of this problem worthy of further consideration. In particular, the apparent lack of relationship between therapeutic effect and plasma level deserves scrutiny.

In a cohort of patients said to have responded to an antidepressant there will be three sub-groups of responders. Some will have responded to the drug's pharmacological effect, some will be placebo responders and some will have undergone a spontaneous remission. It is well known that there is a profound placebo response in depresstve illness (up to $40 \%$ ) and it is well known that the illness tends to wax and wane or remit without apparent cause. Therefore, in any cohort of antidepressant 'responders' it is possible, if not likely, that a majority of the patients will have recovered despite the antidepressant rather than because of it. When the whole group of responders have their antidepressant levels measured, the variation in magnitude is often substantial and no exact 'therapeutic range' can be established. This is hardly surprising considering the possible precipitants of the observed response. It is probable that, if responders to the pharmacological effect of antidepressants could be accurately identified, a therapeutic range could be defined and accepted for many antidepressants. Until 'pharmacological responders' can be identified it is unlikely that any further 


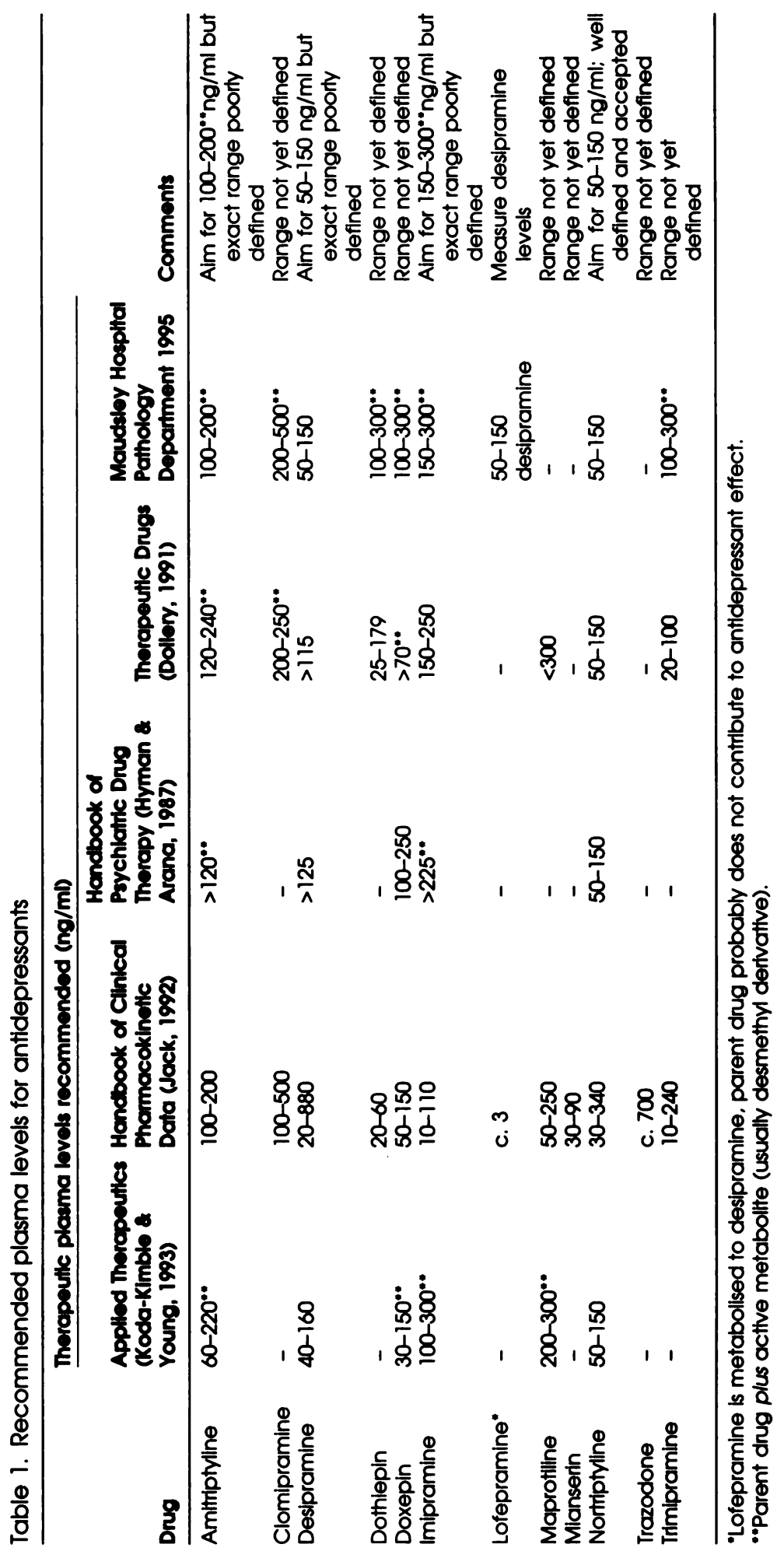


progress in relating plasma level to effect will be forthcoming.

So far, a definite therapeutic range has been established only for nortriptyline. Other drugs have rather more loosely defined target ranges which may assist dosing. Most tricyclic and related compounds have very poorly defined target ranges. In general, tricyclic serum levels should be used only to assure patient compliance or to confirm toxicity due to overdose or adverse interaction.

\section{References}

Douery, C. T. (ed) (1991) Therapeutic Drugs. London: Churchill Livingstone.
Glassman. A. H., Schilkraut, J. J.. Orsulak, P. J.. et al (1985) Tricyclic antidepressant blood level measurements and clinical outcome: an APA task force report. American Journal of Psychiatry, 142. 155-162.

HYMAN, S. E. \& ARANA. G. W. (1987) Handbook of Psychiatric Drug Therapy. Boston, USA: Little, Brown \& Co.

JACK. D. B. (1992) Handbook of Clinical Pharmacokinetic Data. Basingstoke: Macmillan.

KoDA-KimBle, M. A. \& Young, L. Y. (eds) (1992) Applied Therapeutics, 5th ed. Vancouver: Applied Therapeutics.

PRESKORN, S. H. \& FAST, G. A. (1991) Therapeutic drug monitoring for antidepressants: efficacy, safety, and cost effectiveness. Journal of Clinical Psychlatry, 62(Suppl. 6). 23-33.

David Taylor, Principal Pharmacist; and Denise Duncan, Drug Information Pharmacist, The Maudsley Hospital, Denmark Hill, London SE5 8AZ 